\title{
Using modified whole-mount in situ hybridization to study mpo expression in zebrafish
}

\author{
LI-JING SHEN ${ }^{1,2}$, LAN-FANG CAO ${ }^{1}$, FANG-YUAN CHEN ${ }^{2}$, YONG ZHANG ${ }^{2}$, \\ $\mathrm{JI}_{-\mathrm{HUA}} \mathrm{ZHONG}^{2}$ and HUA $\mathrm{ZHONG}^{2}$ \\ Departments of ${ }^{1}$ Pediatrics and ${ }^{2}$ Hematology, Renji Hospital, \\ Shanghai Jiao Tong University School of Medicine, Shanghai 200001, P.R. China
}

Received November 14, 2012; Accepted January 24, 2013

DOI: $10.3892 /$ etm.2013.947

\begin{abstract}
In this study, we cloned the myeloperoxidase (mpo) gene of zebrafish and prepared a digoxigenin-labeled mpo RNA probe to investigate mpo gene expression in zebrafish during embryonic development by whole-mount in situ hybridization (WISH). The earliest mpo expression was detected in cells of the intermediate cell mass (ICM) at $18 \mathrm{~h}$ post-fertilization (hpf). It was detected 1 to $2 \mathrm{~h}$ later in cells in the rostral blood island (RBI) and strong signals were observed in the anterior ICM. Then, it spread over the yolk sac. By 72 hpf these mpo-expressing cells were in the circulation and distributed throughout the embryo. We identified that the level of mpo expression detected by WISH at an early stage was consistent with the data of cytological analyses of adult fish. The use of this method enabled us to track the gene changes that took place before morphological phenotypes were detected, as well to as investigate the hematopoietic cell fate in mutational or transgenic models in vivo. In this study, we modified several steps of WISH. The improved hybridization results demonstrated high specificity, distinct coloration and low background figures.
\end{abstract}

\section{Introduction}

In the last decade, the zebrafish (Danio rerio) was identified as a new genetic system in which to analyze hematopoietic development. Hematopoiesis in zebrafish is similar to that in mammals and other higher vertebrates whose representative blood cell types include the erythroid, thrombocytic, myeloid and lymphoid lineages. In mammals, primitive hematopoiesis occurs outside the embryo, in the blood islands of the yolk

Correspondence to: Professor Lan-Fang Cao, Department of Pediatrics, Renji Hospital, Shanghai Jiao Tong University School of Medicine, 145 Shandong Middle Road, Shanghai 200001, P.R. China E-mail: clf530417@gmail.com

Key words: zebrafish, myeloperoxidase, in situ hybridization, myelopoiesis, modify sac. Later in development, it moves to the aorta-gonadmesonephros (AGM) region, fetal liver and ultimately the bone marrow. By contrast, primitive hematopoietic stem cells (HSCs) in the zebrafish embryo are born intra-embryonically in ventral mesoderm-derived tissue called the intermediate cell mass (ICM). During this wave, the anterior part of the embryo generates myeloid cells, while the posterior part generates mostly erythrocytes and a number of myeloid cells. From $24 \mathrm{~h}$ post-fertilization (hpf), these primitive blood cells start to circulate throughout the embryo. Subsequently, the definitive HSCs emerge from the ventral wall of the dorsal aorta and migrate to the posterior region in the tail called the caudal hematopoietic tissue (CHT). From 3 days post-fertilization (dpf), lymphopoiesis initiates in the thymi. By $4 \mathrm{dpf}$, HSCs seed the kidney marrow, which is equivalent to bone marrow in mammals (1).

Myelopoiesis is the process of producing all types of myeloid cells including granulocytes and monocytes/ macrophages. A number of zebrafish myelopoietic genes are expressed in patterns consistent with their mammalian orthologs, including myeloperoxidase (mpo), an enzyme that is a major component of human neutrophil and eosinophil granules. It is also a marker for zebrafish granulocytes (2). Myeloid cells have a wide spectrum of activities, including immune surveillance and tissue remodeling. Irregularities in myeloid cell development and their function are associated with the onset and the progression of a variety of human disorders, including leukemia (3). An in-depth study of mpo expression and function in zebrafish is likely to improve our ability to identify, isolate and culture hematopoietic cells to enhance our ability to use this simple organism to address disease biology.

Whole-mount in situ hybridization (WISH) is the method of choice to characterize the spatial distribution of gene transcripts during embryonic development. Initial protocols used non-radioactive digoxigenin (DIG)-labeled probes that permit for the first time visualization of global gene expression patterns in Drosophila embryos (4). We present a modified protocol, using a DIG-labeled probe to detect the spatio-temporal spectrum of mpo expression in zebrafish, which reduces the number of steps and obtains signal enhancement. 


\section{Materials and methods}

Animals. The AB zebrafish strain was maintained at $28.5^{\circ} \mathrm{C}$ as described by Westerfield (5). Embryos were staged as described by Kimmel et al (6). Developmental stages refer to hpf or dpf. This study was approved by the Institutional Animal Care and Use Committee (IACUC) of Shanghai Research Center for Model Organisms (Shanghai, China) with approval ID 2012-0008.

Experimental materials. Various restriction enzymes and the TOP10 Escherichia coli strain were purchased from Takara Bio Inc. (Japan). T4-DNA ligase was purchased from Promega Corporation (Madison, WI, USA) and DNA high fidelity polymerase KOD-Plus was purchased from Toyobo Co., Ltd. (Japan). The DIG-deoxyribonucleotide triphosphate (dNTP) labeling kit, blocking reagents, anti-DIG-AP and BM purple were purchased from Roche Diagnostics (Indianapolis, IN, USA). RNase inhibitor was purchased from Ambion (USA), levamisole, heparin and yeast RNA were purchased from Sigma (St. Louis, MO, USA). TRIzol reagent was purchased from Invitrogen Life Technologies (Carlsbad, CA, USA) and the plasmid Maxiprep kit was purchased from Qiagen (Hilden, Germany). The first-strand cDNA Quantscript RT kit was purchased from Tiangen Biotech Co., Ltd. (Beijing, China). A fluorescence microscope (SMZ-1500; Nikon, Japan), ultraviolet spectrophotometer (ASP-3700; ACT Gene, Piscataway, NJ, USA), Biometra $\mathrm{T}$ personal polymerase chain reaction (PCR) amplification instrument (Goettingen, Germany), gel imaging analysis system (Tanon 3500, Shanghai, China) and SPX biochemical incubator (GNP-9160; Shanghai Jinghong Laboratory Instrument Co., Ltd., China) were also used.

Cloning and mpo/pBK-CML plasmid construction. Total RNA was extracted from 40 embryos at $48 \mathrm{hpf}$, using TRIzol reagent according to the manufacturer's instructions. The cDNA was synthesized from $1 \mu \mathrm{g}$ total RNA using the first-strand cDNA Quantscript RT kit. The specific primers were designed according to the zebrafish mpo (zmpo) genomic sequence on the National Center for Biotechnology Information (NCBI) web site (gene ID, 337514); forward primer: 5'-TTCAAGTCCAGAACCAGTGAGCCT-3' and reverse primer: 5'-TTTAGCAGTGGCAGGAAGGATGGA-3'. The length of the amplified PCR product was $2442 \mathrm{bp}$, including two restriction enzyme cutting sites (XhoI at $216 \mathrm{bp}$ and $E c o$ RI at $2384 \mathrm{bp}$ ) and the probe sequence (at $737 \mathrm{bp}$ ). The probe sequence was designed to span exon borders of the gene. PCR was performed as follows: $95^{\circ} \mathrm{C}$ for $10 \mathrm{~min}$; then 35 cycles at $95^{\circ} \mathrm{C}$ for $30 \mathrm{sec}, 58^{\circ} \mathrm{C}$ for $30 \mathrm{sec}$ and $72^{\circ} \mathrm{C}$ for $60 \mathrm{sec}$. The integrity of the PCR product was examined by $1 \%$ agarose gel electrophoresis. The purity was analyzed based on the absorbance ratio at 260 and $280 \mathrm{~nm}$ (A260/280). Then the zmpo fragment and the $\mathrm{pBK}-\mathrm{CML}$ vector were digested with $X h o I$ and EcoRI enzymes and connected at the same sticky end with the T4 ligase, which resulted in the construct of an $\mathrm{mpo} / \mathrm{pBK}-\mathrm{CML}$ plasmid.

Plasmid linearization and probe incubation. The $m p o / p B K-C M L$ plasmid was linearized with the SalI restriction enzyme. DIG-antisense RNA probes were generated by
T7 in vitro transcription ( $1 \mu \mathrm{g}$ linearization template DNA, $1 \mu \mathrm{l}$ DIG-dNTP mix, $2 \mu \mathrm{l} 100 \mathrm{mM}$ dithiothreitol (DTT), $4 \mu \mathrm{l}$ 5X transcription buffer, $1 \mu 1$ RNase inhibitor, $1 \mu 1$ T7 RNA polymerase and diethylpyrocarbonate (DEPC) $\mathrm{H}_{2} \mathrm{O}$ to make $20 \mu \mathrm{l}$, incubated for $2 \mathrm{~h}$ at $37^{\circ} \mathrm{C}$ ). Then, $1 \mu \mathrm{l}$ DNaseI was added and incubated at $37^{\circ} \mathrm{C}$ for $20 \mathrm{~min}$ to purify the product. The final precipitation was stored at $-20^{\circ} \mathrm{C}$.

Embryo preparation. The required developmental stage of the embryos was selected according to the somite. Embryos younger than $48 \mathrm{hpf}$ were dechorionated. The embryos were digested in $1 \mathrm{mg} / \mathrm{ml}$ pronase for $\sim 2-10 \mathrm{~min}$. The digestion was stopped when $>10 \%$ embryos were free from their chorions. The chorions were broken with air from a pipe. If the chorions were difficult to remove, they were manually broken with a pair of tweezers. Embryos older than $24 \mathrm{hpf}$ were decolored in 5\% hydrogen peroxide and $5 \%$ potassium peroxide fish water for $15 \mathrm{~min}$. Embryos (up to 40 embryos per $1.5 \mathrm{ml}$ eppendorf tube) were fixed in fresh $4 \%$ paraformaldehyde (PFA) in phosphatebuffered saline (PBS) and agitated overnight at $4^{\circ} \mathrm{C}$. Then, the embryos were washed twice in PBS with Tween-20 (PBST) for 5 min each and dehydrated with gradient $(25,50,75$ and $100 \%)$ methanal/PBST for 10 min each at room temperature (RT), then stored at $-20^{\circ} \mathrm{C}$.

First day of hybridization. The embryos were removed from the $-20^{\circ} \mathrm{C}$ storage and rehydrated with gradient $(75,50,25$ and $0 \%$ ) methanal/PBST. The embryos were fixed with $4 \%$ PFA for $20 \mathrm{~min}$ and rinsed twice with PBST for $5 \mathrm{~min}$ at RT. The embryos were digested with an appropriate concentration of proteinase $\mathrm{K}\left(10 \mu \mathrm{g} / \mathrm{ml}\right.$ in PBST) at $37^{\circ} \mathrm{C}$ for $3 \mathrm{~min}$ and then fixed and rinsed as above. They were then incubated at $68^{\circ} \mathrm{C}$ in negative hybrid liquid ( $\mathrm{HYB}^{-}, 50 \%$ formamide in $5 \mathrm{X} \mathrm{SSC}$ buffer) for $15 \mathrm{~min}$ and prehybridized at $68^{\circ} \mathrm{C}$ in $300 \mu \mathrm{l}$ positive hybrid liquid $\left(\mathrm{HYB}^{+}, 0.5 \mathrm{mg} / \mathrm{ml}\right.$ yeast RNA and $50 \mu \mathrm{g} / \mathrm{ml}$ heparin in $\mathrm{HYB}^{-}$) for $4 \mathrm{~h}$. Following this, the $\mathrm{HYB}^{+}$was replaced with fresh $\mathrm{HYB}^{+}$containing the DIG-labeled probe (concentration, $\sim 1 \mathrm{ng} / \mu \mathrm{l}$ ) and incubated overnight at $68^{\circ} \mathrm{C}$.

Second day of hybridization. The probe was removed and the embryos were washed twice for $30 \mathrm{~min}$ each with $50 \%$ formamide in $2 \mathrm{X} \mathrm{SSC}$ buffer, then for $15 \mathrm{~min}$ in $2 \mathrm{X} \mathrm{SSC}$ buffer and twice for $30 \mathrm{~min}$ each in $0.2 \mathrm{X}$ SSC buffer at $68^{\circ} \mathrm{C}$. Then, the embryos were rinsed three times for $5 \mathrm{~min}$ each at RT in MABT (100 mM maleic acid, $150 \mathrm{mM} \mathrm{NaCl}$ and $0.1 \%$ Tween-20; $\mathrm{pH} 7.5$ ) and blocked for $1 \mathrm{~h}$ at RT with blocking solution (10\% blocking reagent and $50 \%$ lamb serum in MAB). Anti-DIG-AP (1:4000 dilution in blocking solution) was added and agitated overnight at $4^{\circ} \mathrm{C}$.

Third day of hybridization. The embryos were washed with blocking solution for $30 \mathrm{~min}$ and MABT for $1 \mathrm{~h}$ at RT. Then, the embryos were soaked three times for $5 \mathrm{~min}$ each in staining buffer (100 mM Tris, $50 \mathrm{mM} \mathrm{MgCl} 2,100 \mathrm{mM} \mathrm{NaCl}$, $0.1 \%$ Tween-20 and $1 \mathrm{mM}$ levamisole; $\mathrm{pH}$ 9.5). The embryos were transferred to a 12-well plate, incubated in $1 \mathrm{ml} /$ well BM purple stain for $30 \mathrm{~min}$, monitored with a dissecting microscope every $30 \mathrm{~min}$. The reaction was terminated by being washed twice in PBST and fixed with $4 \%$ PFA at $4^{\circ} \mathrm{C}$ to store. Images were taken in $3 \%$ methyl cellulose. 
A

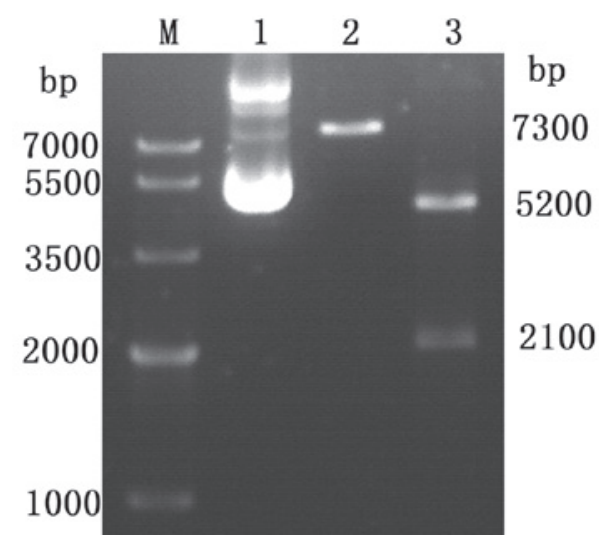

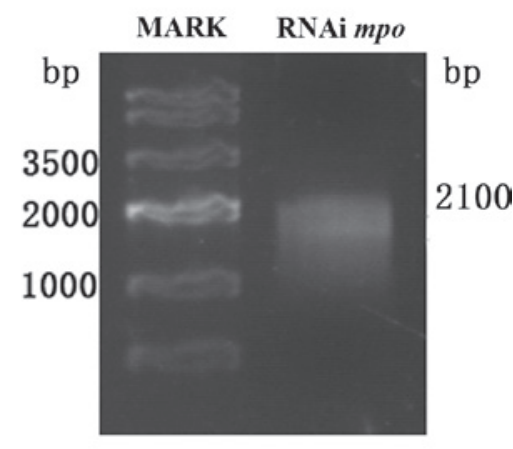

Figure 1. Electrophoresis map of recombinant plasmid and mpo probe. (A) Electrophoresis map of the $m p o / \mathrm{pBK}-\mathrm{CML}$ recombinant plasmid. M, Tiangen marker IV; lane 1, plasmid crude extract. Three bands were observed; relaxed DNA, open circle plasmid and superhelix plasmid; lane 2, the plasmid was linearized by SalI enzyme, which obtained the single band at $7300 \mathrm{bp}$; lane 3, two enzymes, XhoI and EcoRI, obtained two bands at 5200 and $2100 \mathrm{bp}$, respectively, which are the pBK-CML vector and the mpo gene segment. (B) Electrophoresis map of the antisense mpo probe. The band was at 2100 bp. mpo, myeloperoxidase.

\section{Results}

mpo/pBK-CML plasmid construction and probe synthesis. The cDNA of $48 \mathrm{hpf}$ embryos was used as a template to amplify the mpo gemonic fragment by PCR. The PCR product was verified by $1 \%$ agarose gel electrophoresis and the single band at 2400 bp was observed as expected. The PCR product was cloned into the $\mathrm{pBK}-\mathrm{CML}$ carrier and the $m p o / \mathrm{pBK}-\mathrm{CML}$ recombinant plasmid was constructed. The plasmid was linearized by SalI and the single band at $7300 \mathrm{bp}$ was observed. The plasmid was digested with two enzymes (XhoI and EcoRI), producing two bands at 5200 and 2100 bp, respectively, which were the pBK-CML vector and mpo gene segment. This demonstrated that plasmid construction was successful (Fig. 1A). The DIG-labeled antisense mpo mRNA probe was generated by $\mathrm{T} 7$ in vitro transcription and confirmed in the electrophoresis tank soaked with DEPC $\mathrm{H}_{2} \mathrm{O}$ overnight. There was a single band near 2100 bp (Fig. 1B).

Temporal and spatial expression patterns of mpo. The expression patterns of mpo were investigated in zebrafish embryos from 12 to $72 \mathrm{hpf}$ by in situ hybridization using the DIG-labeled antisense RNA probe. As shown in Fig. 2, the earliest expression of zebrafish mpo was detected in cells of the ICM at $18 \mathrm{hpf}$ and 1 to $2 \mathrm{~h}$ later, it was detected in cells in the rostral blood island (RBI). Strong signals were observed in the anterior ICM, then it spread over the yolk sac. By $72 \mathrm{hpf}$ the mpo-expressing cells were in the circulation and distributed throughout the embryo. In our previous study, we established the transgenic enhanced green fluorescent protein $[\operatorname{Tg}(z l y z$ :EGFP)] zebrafish line (7), which expresses EGFP in primitive neutrophils $\left(m p o^{+}\right)$. The EGFP distribution coincided with the result of in situ hybridization of mpo (Fig. 2).

In situ hybridization and cytological analyses. To test whether mpo expression detected by in situ hybridization at an early stage could predict the myelopoiesis in zebrafish development, we compared the peripheral blood smear between wild type (WT) and Tg zebrafish. In our previous study, we established the $\operatorname{Tg}(M Y C N: H S E: E G F P)$ zebrafish line, which suggested that $M Y C N$ converted erythropoiesis to myelopoiesis (Shen et al, the influence of MYCN gene on the transcriptional regulation of hematopoiesis. abs. 751, 9-12 June, 2011). As demonstrated by in situ hybridization, the $M Y C N$ gene increases the expression of mpo in embryos at $22 \mathrm{hpf}$ (Fig. 3A and C). For cytological analyses, blood cells collected from the zebrafish at $60 \mathrm{dpf}$ were transferred onto glass slides using Cytospin, stained with Wright-Giemsa stain and examined under oil immersion by light microscopy (8). This revealed that the blood cells from WT fish were predominantly erythrocytes, with myeloid cells occasionally observed. By contrast, erythrocytes were significantly inhibited in $\mathrm{Tg}$ fish, which were enriched with granulocytes (Fig. 3B and D).

Modified WISH. In order to avoid background staining, unincorporated nucleotides were removed from the probe preparation. We routinely used Ambion NucAway Spin Columns to purify the RNA probe according to the manufacturer's instructions (Cat. AM10070). Following the final precipitation, the hydrolyzed probe was placed in $\mathrm{HYB}^{+}$(final concentration, $\sim 1 \mathrm{ng} / \mu \mathrm{l}$ ) and stored at $-20^{\circ} \mathrm{C}$. Each time the probe was used, it was degenerated in advance by placing it at $95^{\circ} \mathrm{C}$ for $5 \mathrm{~min}$ then on ice for $5 \mathrm{~min}$ to eliminate single RNA spontaneous formation of hairpin structures. Probes were reused for 4-5 times. When 24 hpf embryos were collected, the conventional decolorization method was used and they were placed in $0.03 \%$ phenylthiourea (PTU) in fish water at $12-36 \mathrm{hpf}$. We used the improved decolorizing liquid of $5 \%$ hydrogen peroxide and $5 \%$ potassium peroxide in fish water. As a result, the embryos only required soaking at $24 \mathrm{hpf}$ for $10-15 \mathrm{~min}$. The effect of decolorization was good without damaging the integrity of the embryo. Prior to hybridization, the embryos were permeabilized by digesting with proteinase $\mathrm{K}(10 \mu \mathrm{g} / \mathrm{ml}$ in PBST) at RT for 5-30 min, depending on the developmental stage. The permeability of the probe was appropriate and the staining background was low. In addition, the traditional permeabilization method included digesting the embryos at RT for 5-30 min, depending on the developmental stage. This 
A

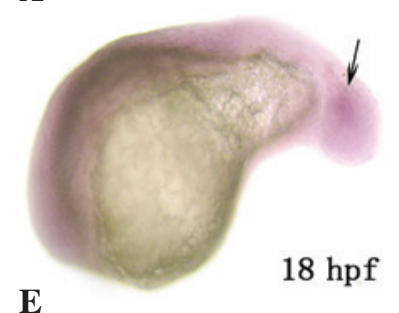

$\mathbf{E}$

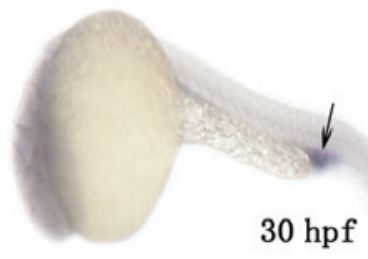

B

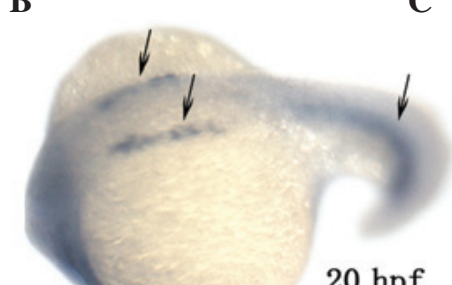

F

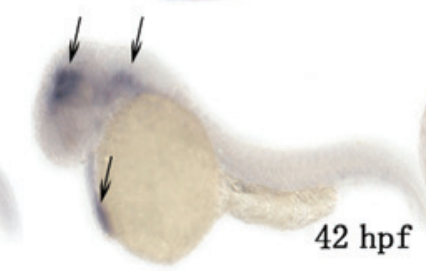

C

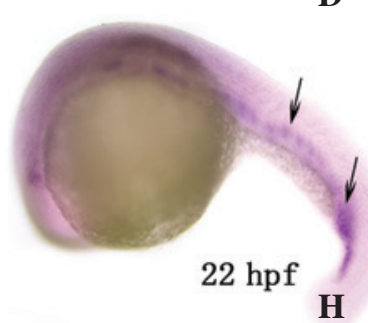

D

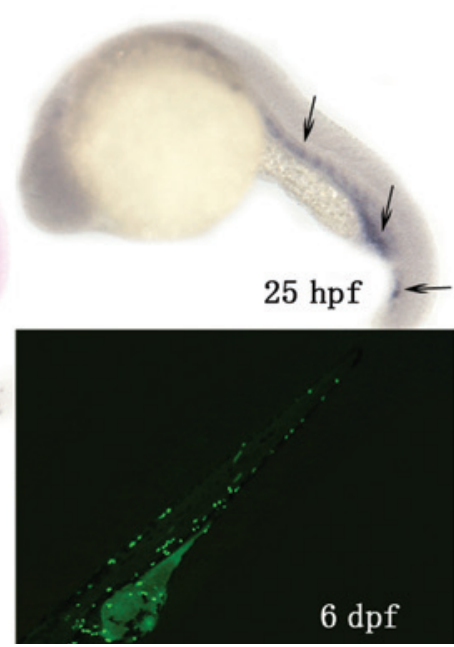

Figure 2. Spatio-temporal spectrum of mpo expression in the AB zebrafish strain. In situ hybridization with a digoxigenin-labeled RNA probe was used to detect zebrafish mpo expression in embryos at $18 \mathrm{hpf}$ (A), $20 \mathrm{hpf}$ (B), $22 \mathrm{hpf}$ (C), $25 \mathrm{hpf}$ (D), $30 \mathrm{hpf}$ (E), $42 \mathrm{hpf}$ (F) and $72 \mathrm{hpf}$ (G). Black arrows indicate $m p o$ expression in the intermediate cell mass (ICM) (A-E), rostral blood island (RBI) and pericardial cavity (F). At 72 hpf these mpo-expressing cells were in the circulation and distributed throughout the embryo $(\mathrm{G})$. (H) Distribution of fluorescent neutrophils $\left(m p o^{+}\right)$in our previously established $\mathrm{Tg}(z l y z$ :EGFP) embryo at 6 dpf. mpo, myeloperoxidase; EGFP, enhanced green fluorescent protein; hpf, hours post-fertilization; dpf, days post-fertilization.

A

WT

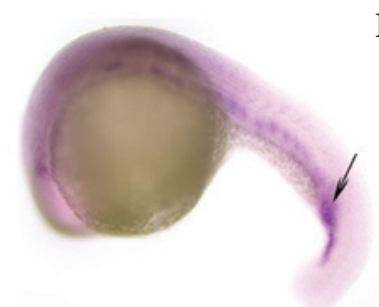

B

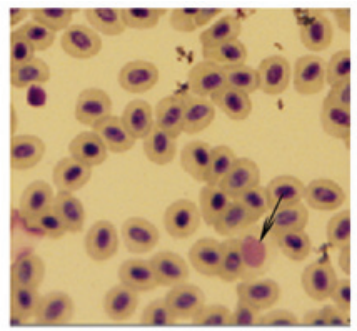

C

TG
D

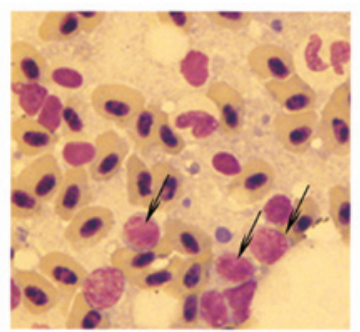

Figure 3. In situ hybridization of mpo expression and peripheral blood smear comparison between wild type and $\operatorname{Tg}(M Y C N$ :HSE:EGFP) zebrafish. In situ hybridization with a digoxigenin-labeled RNA probe to detect wild type (WT) (A) and our previously established $\operatorname{Tg}(M Y C N$ :HSE:EGFP) (TG) (C) zebrafish mpo expression in embryos at $22 \mathrm{hpf}$. Black arrows indicate greater mpo expression in TG zebrafish than in WT embryos. Cytological analyses of hematopoietic cells from WT (B) and TG (D) zebrafish at $60 \mathrm{dpf}$. Blood cells from WT fish were predominantly erythrocytes, with myeloid cells occasionally observed. By contrast, erythrocytes were significantly inhibited in TG fish, which were enriched with granulocytes (indicated by black arrows). mpo, myeloperoxidase; EGFP, enhanced green fluorescent protein; TG, transgenic; hpf, hours post-fertilization; HSE, heat shock elements.

is difficult to control. We improved the digestion temperature to $37^{\circ} \mathrm{C}$ and the process was shorted to $5 \mathrm{~min}$.

\section{Discussion}

The zebrafish system has a number of unique advantages compared to other vertebrate model organisms. The embryos

are externally fertilized and transparent, enabling in vivo visualization of early embryonic processes ranging from birth of HSCs in the mesoderm to migration of blood cells. In addition, large production of embryos makes phenotype-based forward genetics feasible (9). A steadily increasing number of hematopoietic-specific genes have been cloned in zebrafish, providing molecular reagents and markers for specific stages of hematopoietic differentiation and specific cell types. We constructed the DIG-labeled mpo RNA probe to investigate mpo gene expression in zebrafish embryos. The earliest mpo expression was detected in cells of the ICM at $18 \mathrm{hpf}$ and 1-2 h later, it was detected in cells in the RBI. Strong signals were observed in the anterior ICM, then it spread over the yolk sac. By 72 hpf the mpo-expressing cells were in the circulation and distributed throughout the embryo, with a tendency for a subpopulation of mpo-expressing cells to be aggregated in the ventral vein region. Later-stage expression was difficult to distinguish by WISH.

We identified that the level of mpo expression detected by WISH at an early stage was consistent with the result of cytological analyses of adult fish (Fig. 3). This method enabled us to track the gene changes that took place before morphological phenotypes were detected, as well as investigate the hematopoietic cell fate in mutational or transgenic models in vivo. Given the considerable morphological and functional parallels between zebrafish and mammalian myeloid cells, it is not surprising that zebrafish show conservation of the molecular regulation of myelopoiesis and the molecular tools for myeloid cell function (3).

In this study, we modified several steps of WISH. We carried out several pretreatment steps to ensure the purity and concentration of the probe, as well as shorten the digestion time by proteinase $\mathrm{K}$ at higher temperatures. In the decolorization step, we avoided using PTU, which simplified the zebrafish embryo culture steps and enhanced the environmental protection. Finally, we selected BM purple dye to simplify the staining step. The improved hybridization results 
demonstrated high specificity, distinct coloration and low background figures.

Using WISH in zebrafish, we have the ability to identify and study the lesions of myelopoiesis. Therefore, the powerful genetic approaches applicable in this model, the genomic resources being collected by the international zebrafish and genomic communities and the ability to study myeloid development in this model organism provide new insights into the myeloid arm of developmental hematology.

\section{Acknowledgements}

The authors thank all members of the Shanghai Research Center for Biomodel Organisms and the Shanghai Institute of Hematology for excellent technical support. This study was supported in part by the National Natural Science Foundation of China Grant (30900636), the Science and Technology Commission of Shanghai Municipality Grant (08JCl414900), the Science and Technology Commission of Shanghai Fund Project of the TCM Guide Project (12401906700) and the Science and Technology Fund Project of Shanghai Jiaotong University School of Medicine (09XJ21066).

\section{References}

1. Paik EJ and Zon LI: Hematopoietic development in the zebrafish. Int J Dev Biol 54: 1127-1137, 2010

2. Xu J, Du L and Wen Z: Myelopoiesis during zebrafish early development. J Genet Genomics 39: 435-442, 2012.

3. Forrester AM, Berman JN and Payne EM: Myelopoiesis and myeloid leukaemogenesis in the zebrafish. Adv Hematol 2012: 358518, 2012.

4. Tautz D and Pfeifle C: A non-radioactive in situ hybridization method for the localization of specific RNAs in Drosophila embryos reveals translational control of the segmentation gene hunchback. Chromosoma 98: 81-85, 1989.

5. Westerfield M (ed): The Zebrafish Book: A Guide for the Laboratory Use of Zebrafish (Danio rerio). University of Oregon Press, Eugene, pp1.1-1.27, 1995.

6. Kimmel CB, Ballard WW, Kimmel SR, Ullmann B and Schilling TF: Stages of embryonic development of the zebrafish. Dev Dyn 203: 253-310, 1995.

7. Zhang Y, Bai XT, Zhu KY, et al: In vivo interstitial migration of primitive macrophages mediated by JNK-matrix metalloproteinase 13 signaling in response to acute injury. J Immunol 181: 2155-2164, 2008

8. Yeh JR, Munson KM, Chao YL, Peterson QP, Macrae CA and Peterson RT: AML1-ETO reprograms hematopoietic cell fate by downregulating scl expression. Development 135: 401-410, 2008.

9. Shafizadeh E and Paw BH: Zebrafish as a model of human hematologic disorders. Curr Opin Hematol 11: 255-261, 2004. 